\title{
Contribución de Vigna unguiculata L. a la sustentabilidad de sistemas de cultivo de caña de azúcar
}

\section{Contribution of Vigna unguiculata L. to the sustainability of systems for the cultivation of sugar cane}

\section{Vigna unguiculata L. contributo para a sustentabilidade dos sistemas de produção de cana}

\author{
Oscar Eduardo Sanclemente Reyes ${ }^{1}$, Milton Cesar Ararát Orozco으 \& Cristihan Andrés De la cruz Cardona ${ }^{3}$ \\ 'Ingeniero Ambiental, Especialista en Pedagogía, Magister en Ciencias Agrarias, Doctor en \\ Agroecología. ${ }^{2}$ Ingeniero Agrónomo, Magister y Doctor en Ciencias Agrarias. ${ }^{3}$ Agrónomo.

\begin{abstract}
1, 2Programa de Ingeniería Ambiental. Escuela de Ciencias Agrícolas, Pecuarias y del Medio Ambiente ECAPMA. Universidad Nacional Abierta y a Distancia UNAD. Palmira. Valle. Colombia. ${ }^{3}$ Gerencia de Campo. Supervisor de Campo. Ingenio Azucarero Riopaila Castilla. Bugalagrande. Valle. Colombia
\end{abstract} \\ 10sedusancle20@hotmail.com, ${ }^{2}$ milton.ararat@hotmail.com, ${ }^{3}$ cristihan.agro@hotmail.com
}

\section{Resumen}

Con el objetivo de evaluar la contribución del abono verde (AV) Vigna unguiculata L., a la producción sostenible de caña de azúcar (CA) Saccharum officinarum L. Var. CC 85-92 en un Vertisol del Valle del Cauca (Colombia), se usó diseño experimental en bloques completos al azar y cinco repeticiones. Los tratamientos fueron: (C) Control- soca de CA, (AV1) siembra 1X1 AV- CA, (AV2) siembra 2X1 AV- CA, (AV1+R) siembra 1X1 AV inoculada con Rhizobium sp.- CA, y (AV2+R) siembra 2X1 AV inoculada con Rhizobium sp.- CA. Se evaluó el aporte de biomasa del $A V$, la biomasa de arvenses asociadas y la humedad del suelo, durante el mes dos. En maduración de CA (mes once) se evaluó la concentración de sólidos y sacarosa en tallos. En cosecha de CA (mes trece) se evaluaron las toneladas de caña por hectárea (TCH), el rendimiento y las toneladas de azúcar por hectárea (TAH), como efecto de los tratamientos. Se observó que el inóculo Rhizobium sp., no tuvo efectos aparentes, por baja especificidad simbiótica con el AV. El AV2, registró aporte significativo $(p<0.05)$ de materia seca (14.5 $\left.\mathrm{tha}^{-1}\right)$ incidiendo en reducción de arvenses y retención de humedad $\left(161 \mathrm{~m}^{3}\right.$ ha-1), frente al C. El AV1 registró productividad en TCH (131.5 t ha-1) y TAH (15.5 t ha-1), significativamente $(p<0.05)$ altas con respecto a los demás tratamientos. Estos resultados muestran las bondades del uso de sistemas intercalados AV- caña de azúcar, sobre la economía y sustentabilidad del sector.

Palabras clave: biomasa, sustentabilidad, economía de cultivo, cambio climático.

\section{Abstract}

With the objective of evaluating the contribution of green manure (AV) Vigna unguiculata L., to the sustainable production of sugarcane (CA) Saccharum officinarum L. Var. CC 85-92 in a Vertisol from the Valle del Cauca (Colombia), an experiment with five repetitions and completely randomized block ex- 
perimental design was used. The treatments were: (C) Control - CA, (AV1) soca seeding $1 \mathrm{X} 1 \mathrm{AV}$ - CA, (AV2) seeding $2 X 1$ AV - CA, (AV1) seeding $1 X 1$ AV inoculated with Rhizobium sp-CA, and (AV2 R) seeding 2 X 1 AV inoculated with Rhizobium sp-CA. Three variables were evaluated during the second month: contribution of AV biomass, weeds associated biomass and moisture of the soil. During maturation of CA (month 11) the concentration of solids and sucrose on stems were evaluated. In the harvest stage of CA (13 month) tons of cane per hectare (TCH), performance and tons of sugar per hectare (TAH were evaluated), as an effect of the treatments. It was observed that the Rhizobium inoculum SP., had no apparent effects, due to low symbiotic specificity with the AV. The AV2, showed significant contribution $(p<$ $0.05)$ for dry matter (14.5 $\left.\mathrm{t} \mathrm{ha}^{-1}\right)$ on reduction of weed and moisture retention (161 $\left.\mathrm{m}^{3} \mathrm{ha}^{-1}\right)$, in comparison to $\mathrm{C}$. The AV1showed significantly higher productivity measured in TCH (131.5 $\left.\mathrm{t} \mathrm{ha}^{-1}\right)$ and TAH (15.5 $\left.t \mathrm{ha}^{-1}\right),(\mathrm{p}<0.05)$ compared to the other treatments. These results showed the benefits of the use of intercropping systems AV - sugar cane, on the economy and sustainability of the sector.

Key-words: biomass, sustainability, economy of cultivation, climate change.

\section{Resumo}

Com o objetivo de avaliar a contribuição do adubo verde (AV) Vigna unguiculata $L$. na produção sustenível da cana de açucar (CA) Saccharum officinarum L. Var. CC 85-92 em um Vertisol do Vale do Cauca (Colômbia) foi estabelecido um delineamento experimental em blocos inteiramente casualizados com cinco repetições, sendo que os tratamentos foram: (C), Control-soca de CA; (AV1), semeadura 1X1 AV-CA; (AV2), semeadura 2X1 AV-CA; (AV1+R), semeadura 1X1 AV inoculada com Rhizobium sp.- CA; (AV2+R), semeadura 2X1 AV inoculada con Rhizobium sp.- CA. Avaliou-se o aporte de biomassa do AV, a biomassa de arvenses associadas e a umidade do solo no mês dois. Na maturidade de CA (mês onze), avaliou-se a concentração de sólidos e sacarose nos caules. Em colheita de CA (mês treze) se avaliaram as toneladas de cana por hectarea $(\mathrm{TCH})$, produtividade e as toneladas de açúcar por hectarea (TAH) como efeito dos tratamentos. Constatou-se que o inoculo Rhizobium SP., não teve efeitos aparentes pela baixa especificidades simbiótica com o tratamento AV. O tratamento AV2 registrou aporte significativo $(p<0.05)$ de materia seca (14.5 t ha-1), impactando na redução de arvenses e na retenção de umidade (161 m3 ha-1) em relação ao tratamento C. O tratamento AV1 registrou productividade em TCH (131.5 t ha-1) e TAH (15.5 t ha-1) significativamente altas $(p<0.05)$ em contraste com os demais tratamentos. Estes resultados mostram as bondades do uso de sistemas intercalados AV- cana de açúcar sobre a economía e sustentabilidade do setor.

Palavras-chave: biomassa, sustentabilidade, economia, economía da cultura, mudança climática.

\section{Introducción}

La producción industrial de caña de azúcar Saccharum offcinarum L. en Colombia, se concentra en cerca de 230.000 ha en los departamentos de Valle del Cauca, Cauca, Nariño y Risaralda (ASOCAÑA, 2008). Este sistema de producción se caracteriza por monocultivos extensos manejados convencionalmente. En las últimas décadas, la productividad del cultivo en toneladas de caña por hectárea $(\mathrm{TCH})$ se ha incrementado sustancialmente, debido principalmente a factores como: desarrollo de variedades adaptadas a condiciones agroecológicas específicas, implementación de agricultura específica por sitio y uso de sistemas de monitoreo y seguimiento.

Sin embargo, se han reportado externalidades en el sistema como: compactación, salinización, sodización y pérdida de la materia orgánica del suelo; 
debidos al continuo uso de maquinaria, sistemas de irrigación ineficientes, aplicación de agroquímicos y quema de caña en cosecha, que afectan su sostenibilidad (Dávalos, 2007; Franco, Torres \& Patoja, 2009; Zúñiga, Osorio, Cuero \& Peña, 2011; Pérez, Peña \& Álvarez, 2011; Cuero, 2012).

Por consiguiente, uno de los desafíos del sector azucarero es el desarrollo de tecnologías alternativas de bajo costo para el mejorar la fertilidad de los cultivos, al tiempo que reduzcan los impactos ambientales sobre el suelo y los recursos hídricos. Una de estas alternativas son los abonos verdes $\mathrm{AV}$, que consisten en la siembra en intercalado o rotación de especies leguminosas, que por fijación del $\mathrm{N}_{2}$ atmosférico suministran cantidades considerables de éste y otros nutrientes al suelo, vía aporte de materia orgánica de rápida descomposición (Da Costa et al., 1993; Prager et al., 2012).

El frijol caupí Vigna unguiculata L., es bastante usado como AV en asocio con gramíneas de interés económico, gracias a su alta producción de biomasa, capacidad adaptativa el medio, rápido crecimiento y alta fijación de $\mathrm{N}_{2}$ por simbiosis con rizobios del suelo (Gana \& Busari, 2001; Obando, 2012; Sanclemente, 2013). En el cultivo de cañas orgánicas, el uso de frijol caupí viene tomando cada vez más auge entre los productores del departamento. El objetivo de este estudio fue evaluar la contribución del AV Vigna unguiculata L., a la producción sostenible de caña de azúcar Saccharum officinarum L. Variedad CC 85-92, en un Vertisol del Valle del Cauca (Colombia).

\section{Materiales y métodos}

\section{Localización y características iniciales del suelo}

El ensayo se realizó durante el año 2015 en la Hacienda Lucernita, jurisdicción del municipio de Bugalagrande (Valle del Cauca). El suelo clasificado como Vertisol, presentó textura Arcillosa, densidad aparente $\left(1.4 \mathrm{~g} \mathrm{~cm}^{-3}\right)$, $\mathrm{pH}(7.4)$, M.O. (1.72\%),
$\mathrm{N}$-total $(0.08 \%), \mathrm{P}\left(8.3 \mathrm{mg} \mathrm{kg}^{-1}\right), \mathrm{K}(0.3 \mathrm{meq} 100 \mathrm{~g}$ $\left.{ }^{1}\right)$, Ca $\left(20.6\right.$ meq $\left.100 \mathrm{~g}^{-1}\right)$ y $\mathrm{Mg}\left(6.2 \mathrm{meq} 100 \mathrm{~g}^{-1}\right)$ Antes del ensayo, el suelo estuvo sembrado por seis años con la variedad de caña CC 85- 92 de CENICAÑA (Centro de Investigación de la Caña de Azúcar. Valle del Cauca. Colombia).

\section{Establecimiento del ensayo en campo}

El suelo se preparó mecánicamente, usando subsolador de cinco brazos y Minitandem, a 25 y $45 \mathrm{~cm}$ de profundidad, respectivamente. Se realizaron dos pases con discos de arado para acomodar la semilla de caña de azúcar. Mediante diseño experimental en bloques completos al azar y cinco repeticiones, se establecieron los siguientes tratamientos: $(C)$ Control- soca de caña de azúcar, (AV1) siembra de frijol caupí intercalado en surcos $1 \mathrm{X} 1$ con soca de caña de azúcar, (AV2) siembra de frijol caupí intercalado en surcos $2 X 1$ con soca de caña de azúcar, (AV1+R) siembra de frijol caupí intercalado en surcos 1X1 con soca de caña de azúcar más inóculo Rhizobium sp., (AV2+R) siembra de frijol caupí intercalado en surcos 2X1 con soca de caña de azúcar más inóculo Rhizobium sp. Los tratamientos se distribuyeron aleatoriamente en parcelas de 31.2 $\mathrm{m}^{2}(19.0 \times 1.65 \mathrm{~m})$.

En $A V 1+R$ y $A V 2+R$, las semillas de frijol caupí se embebieron durante 15 minutos en solución al $1 \%$ del producto comercial Rhizobacter (Rhizobium sp.). Posteriormente, las semillas embebidas se secaron a la sombra durante 2 horas, para garantizar la inoculación de la cepa. La siembra del frijol caupí se realizó con máquina sembradora de cadena de 4 tarros, que aportaron entre 25 y 28 semillas $\mathrm{m}^{-1}$. Se aplicó lámina de 30 $\mathrm{mm}$ de riego de germinación después de siembra, empleando cañones de aspersión. Luego de 15 días, se reinocularon las parcelas $A V 1+R$ y AV2+R con solución Rhizobacter al $1 \%$, usando bomba de aspersión manual. Las labores de riego durante el ensayo, se realizaron tomando como referencia el software balance hídrico de CENICAÑA, buscando mantener la humedad del suelo a capacidad de campo. 


\section{Evaluación de biomasa del frijol caupí y arvenses acompañantes}

Transcurridos 48 días de la siembra, se colectó la biomasa de frijol caupí y arvenses acompañantes, empleando la técnica del cuadrante $(0.5 \times 0.5 \mathrm{~m})$ descrita por López, Fontenot \& García (2011). Se tomaron diez submuestras al azar dentro de las parcelas, incluyendo tallos, ramas y hojas. La biomasa colectada dentro del cuadrante, se secó al aire durante 10 días y posteriormente, se evaluó su materia seca mediante balanza digital. Los valores de materia seca se extrapolaron a ha.

\section{Evaluación de la humedad del suelo}

Simultáneamente a la evaluación de biomasa, se tomaron muestras de suelo a $0-10 \mathrm{~cm}$ de profundidad en cada parcela experimental, para estimar la retención de humedad por gravimetría, como lo describe Jaramillo (2002). Inicialmente, la masa de suelo húmedo se cuantificó en campo, posteriormente, las muestras se llevaron al laboratorio donde se secaron al horno a $105^{\circ} \mathrm{C}$ durante 24 horas. Finalmente, se cuantificó la masa de suelo seco y la humedad gravimétrica.

Evaluación de variables de interés económico en caña de azúcar- Variedad CC 85-92

Durante el mes once posterior a la siembra, se evaluó en campo el contenido de sólidos solubles en los jugos de caña de azúcar. Para ello, se tomaron diez plantas al azar dentro de las parcelas experimentales y se midió en sus entrenudos el contenido de sólidos solubles (\% brix), mediante uso de refractómetro. Adicionalmente, se evaluó en los jugos el contenido de sacarosa, por método polarimétrico según lo descrito por Herrera (2011). La pureza se estimó como la razón porcentual entre el contenido de sacarosa y los grados brix de los jugos.

Al mes trece se cosecharon los tallos de caña de azúcar, descartando los bordes en cada parcela y extrapolando la productividad a $\mathrm{TCH}$. El rendimiento se estimó como la razón porcentual entre las toneladas de azúcar por ha (TAH) (Adaptado de datos de producción final de azúcares, suministrados por el ingenio azucarero Rio Paila- Castilla S.A. Valle del Cauca) y las TCH cosechadas.

\section{Análisis estadístico de los datos}

A los resultados se les realizó Análisis de Varianza ANDEVA y comparación de medias de TUKEY $(p<0.05)$, usando el paquete estadístico SAS versión 9.2 (2008).

\section{Resultados y discusión}

\section{Biomasa de frijol caupí}

El aporte de biomasa en prefloración de frijol caupí, en aquellos tratamientos donde se sembró la especie, se muestra en la Figura 1. El análisis de varianza para datos de biomasa, registró diferencias entre tratamientos. De acuerdo con la prueba de medias de Tukey, AV2 obtuvo $4.5 \mathrm{t} \mathrm{ha}^{-1}$ de materia seca, significativamente $(p<0.05)$ mayor a las demás. Este registro fue similar a los obtenidos en otras investigaciones (Duarte et al., 1995; Padilla, Colón \& díaz, 2001; Shindoi, Prause \& Jover, 2012) donde el frijol caupí fue usado como AV, aportando $\mathrm{C}$ humificable y nutrientes como $\mathrm{N}, \mathrm{P}$, $\mathrm{K}, \mathrm{Ca}$, entre otros.

La aplicación de cepa Rhizobium sp., tanto en $A V 1+R$ y $A V 2+R$ no incidió positivamente sobre incrementos en la materia seca de caupí. De acuerdo a Obando (2012), la simbiosis efectiva rizobio- frijol Caupí, depende de la afinidad génetica entre la especie y la planta, encontrándose dentro del mismo género Rhizobium sp., gran diversidad de especies. Por su parte, Delgado, Casella \& Bedmar (2007) registraron que la aplicación de Rhizobium sullae y Rhizobium etli en suelos con bajos contenidos de materia orgánica, generaron inmovilización y desnitrificación microbiana, sin lograr simbiosis efectiva con Caupí para fijación de $\mathrm{N}_{2}$. En la presente investigación, el frijol caupí logró desarrollar simbiosis con rizobios nativos del suelo.

La densidad de siembra, fue otra variable que incidió sobre la producción de materia seca en caupí, que se incrementó hasta 53\% cuando se duplicó el número de surcos en AV2, indicando mayor beneficio sobre aportes de materia orgánica al suelo, con posterior mineralización y disponibilidad de nutrientes. 


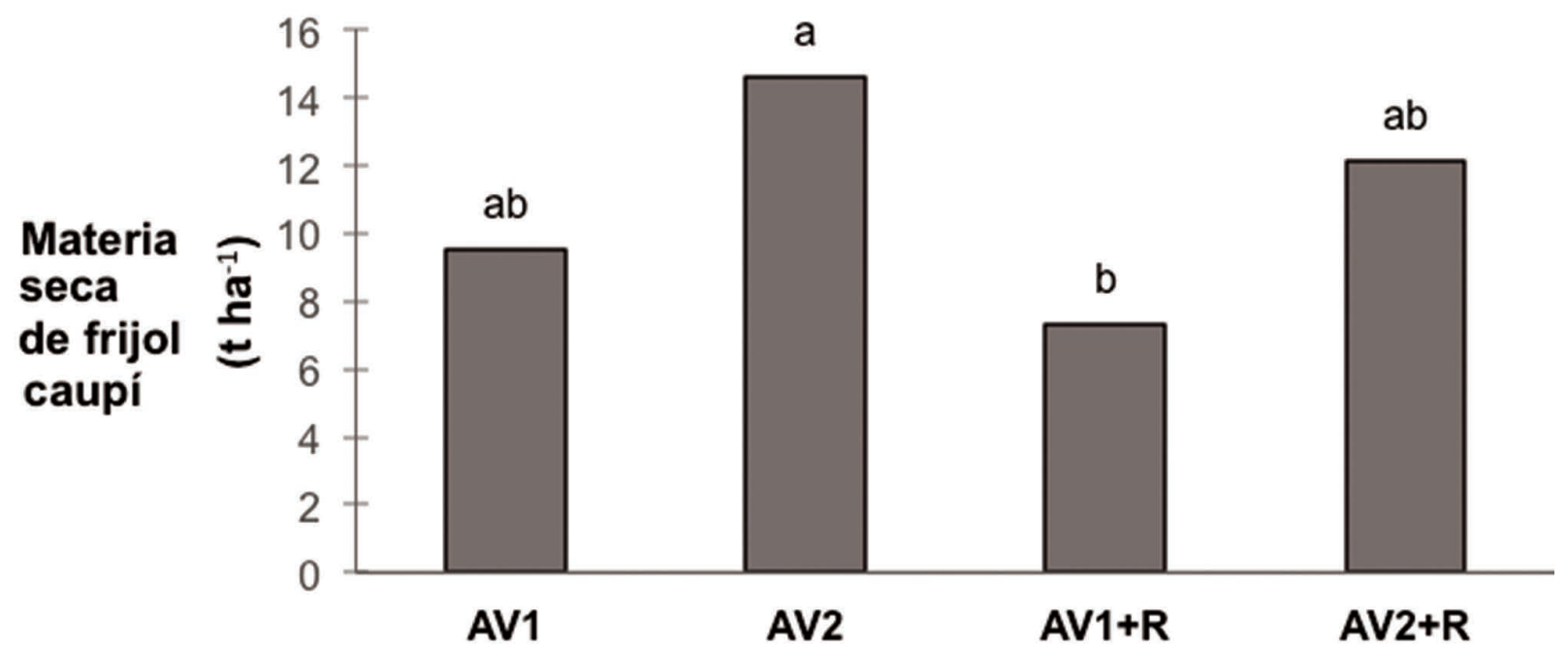

Figura 1. Producción de biomasa de frijol caupí (t ha-1) de materia seca, para los tratamientos evaluados. Se realizó prueba de Tukey $(p<0.005)$.

\section{Biomasa de arvenses acompañantes}

En el cultivo de caña de azúcar, la presencia de arvenses afecta su desarrollo durante los primeros meses por interferencia de luz y nutrientes, siendo factor negativo en productividad. En este ensayo, el análisis de varianza para biomasa de arvenses, registró diferencias significativas $(p<0.05)$ entre tratamientos. De acuerdo con la prueba de medias de Tukey, el tratamiento de control (C) obtuvo $12.7 \mathrm{t} \mathrm{ha}^{-1}$ de materia seca, significativamente $(p<0.05)$ mayor a los demás, como se muestra en la Figura 2.

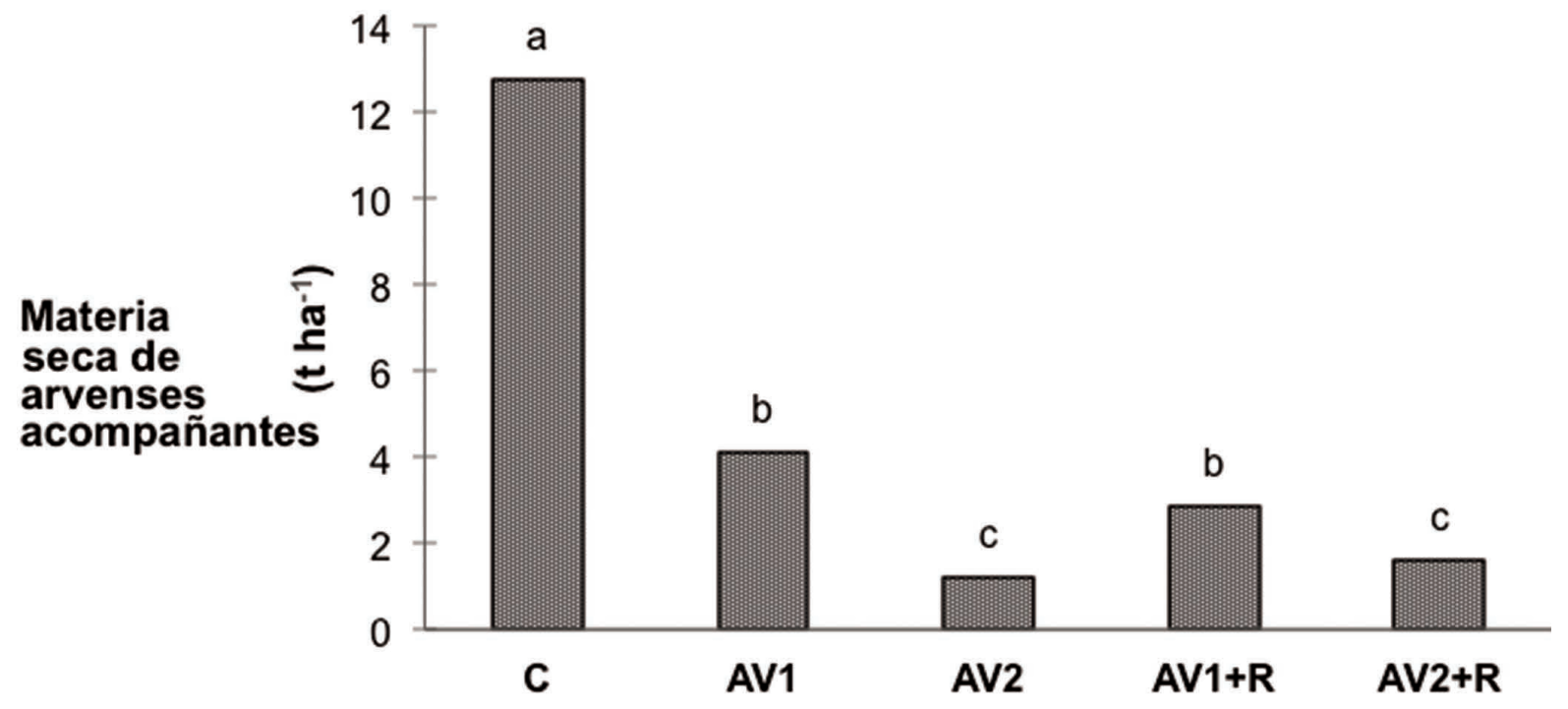

Figura 2. Producción de biomasa de arvenses $\left(\mathrm{t} \mathrm{ha}^{-1}\right)$ de materia seca, en los tratamientos evaluados. Se realizó prueba de Tukey $(p<0.005)$. 
El suelo desnudo en el tratamiento de control, posibilitó la geminación de plantas acompañantes del cultivo de caña de azúcar, que tuvieron condiciones óptimas como disponibilidad de luz, agua y nutrientes del suelo para su desarrollo. En contraste, la siembra de caupí como AV en los demás tratamientos, redujo notablemente las posibilidades de germinación y desarrollo de arvenses. El rápido desarrollo del caupí, actúa como agente supresor de arvenses acompañantes sobre todo de hoja angosta, dada la exigencia de luz de las gramíneas (Berlingeri, Yuncosa \& Pérez, 2008).

Registros históricos de la Hacienda Lucernita, indican la presencia de gran variedad de arvenses pertenecientes a la familia de las gramíneas como: Cyperus rotundus, Cyperus ferax, Rottboelia exaltata, Sorghum halepense, entre otras, que acompañan el cultivo de caña de azúcar. Por su parte, Gómez (1995), reporta alta persistencia de éstas y otras especies de gramíneas en suelos cultivados con caña de azúcar en el departamento del Valle del Cauca. En esta investigación, la siembra 2X1 de caña con caupí, redujo notoriamente las arvenses acompañantes por su alta producción de biomasa, como se ilustró en la Figura 1.

La supresión de arvenses con el uso de caupí como AV en el cultivo de caña de azúcar, es un factor ambientalmente positivo debido a la posibilidad de reducir ostensiblemente la aplicación de herbicidas pre y pos-emergentes, que contaminan el suelo y los recursos hídricos de la región.

\section{Humedad del suelo}

Uno de los factores más importantes para el desarrollo del cultivo de caña de azúcar, es la disponibilidad de agua en el suelo. En este ensayo, el análisis de varianza para datos de humedad gravimétrica del suelo no marcó diferencias significativas $(p<0.05)$ entre tratamientos (Figura 3 ).

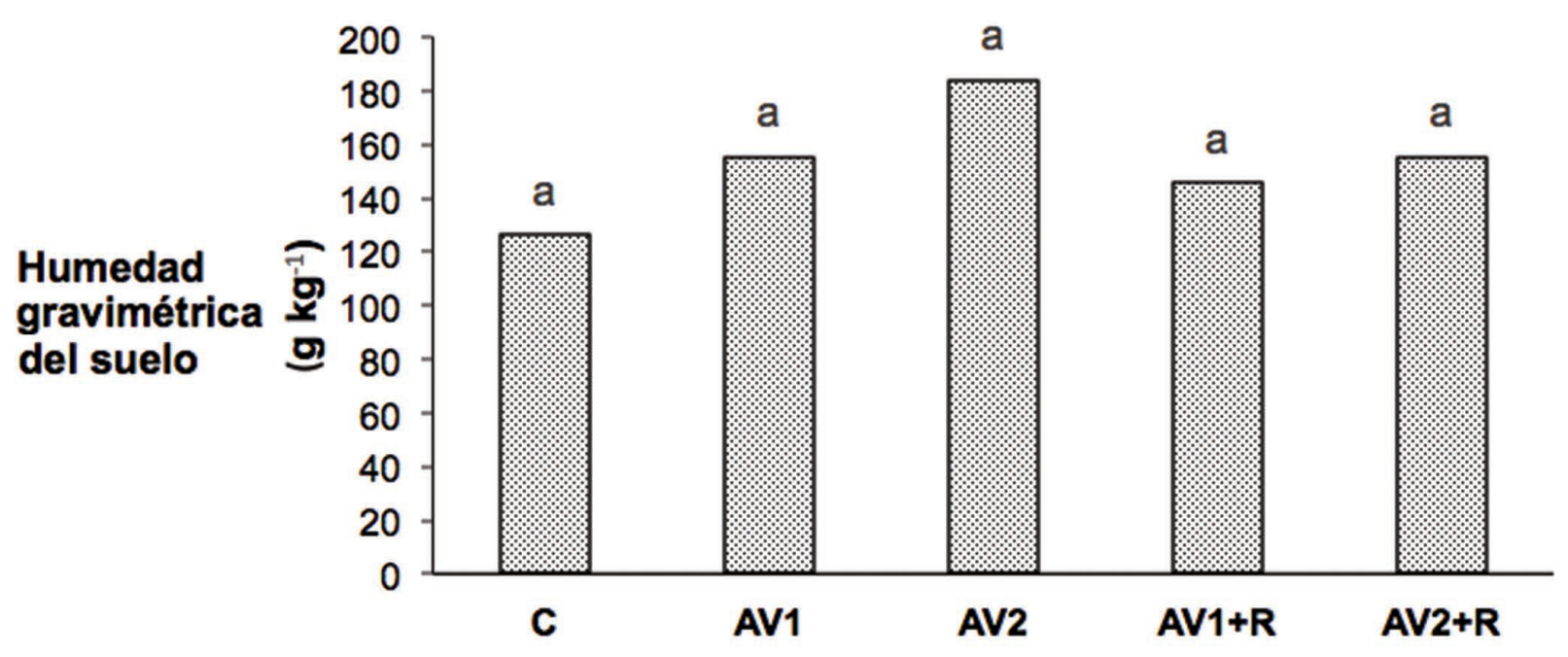

Figura 3. Humedad gravimétrica del suelo $\left(\mathrm{g} \mathrm{kg}^{-1}\right)$ en todos los tratamientos evaluados. Se realizó prueba de Tukey $(p<0.005)$.

Esto indica que tanto los AV como el tratamiento control se comportaron de manera similar para esta variable. A pesar de esto, existieron valores altos de humedad gravimétrica en AV2 que registró $184 \mathrm{~g} \mathrm{~kg}^{-1}$. En términos de lámina de agua, la diferencia entre AV2 y C fue de $16.1 \mathrm{~mm}$, 
equivalentes a $161 \mathrm{~m}^{3} \mathrm{ha}^{-1}$. Este valor es importante, considerando los requerimientos hídricos del cultivo de caña de azúcar, que oscilan entre 1200 y $1500 \mathrm{~mm}$ año-1 (CENICAÑA, 1995).

En el departamento del Valle del Cauca (Colombia), las temporadas secas en suelos arcillosos como el caso de estudio, generan agrietamientos por expansión en superficie de arcillas 2:1, afectando notablemente sus propiedades mecánicas y dejándo el suelo expuesto a la erosión. De aquí la importancia de mantener el suelo protegido con vegetación de cobertura, sobre todo de hoja ancha. Prager et al. (2012) resaltan las ventajas comparativas del uso de AV para proteger el suelo de la erosión y retener la humedad, siendo alternativa viable ante eventos de cambio climático.

\section{Productividad en caña de azúcar}

Los datos de productividad de caña de azúcar se presentan en la Tabla 1. La concentración de sólidos (\% brix) en jugos, fluctuó entre 18.7 y $19.0 \%$ en los tratamientos, sin registrarse diferencias significativas $(p<0.05)$ entre ellos. Del mismo modo, la concentración de sacarosa estuvo entre 17.4 y $18.1 \%$, sin diferencias estadísticas. Estos valores fueron similares a los registrados por Larrahondo \& Villegas (1995), en variedades comerciales de caña de azúcar en el departamento del Valle del Cauca. Aparentemente, la concentración de sólidos y sacarosa en los jugos de caña de azúcar, son afectadas por la siembra intercalada de frijol caupí como AV.

Tabla 1. Productividad de tallos de caña de azúcar Var. CC 85-92, en los tratamientos evaluados

\begin{tabular}{|c|c|c|c|c|c|c|}
\hline $\begin{array}{l}\text { Tratamien- } \\
\text { to }\end{array}$ & $\begin{array}{l}\text { Sólidos* } \\
\text { Brix (\%) }\end{array}$ & $\begin{array}{c}\text { Sacarosa* } \\
(\%)\end{array}$ & $\begin{array}{c}\text { Pureza* }^{*}(\%) \\
\text { (\%) }\end{array}$ & $\begin{array}{l}\mathrm{TCH}^{\star *} \\
\left(\mathrm{t} \mathrm{ha}^{-1}\right)\end{array}$ & $\begin{array}{c}\text { Rendimien- } \\
\text { to }^{\star *}(\%)\end{array}$ & $\begin{array}{l}\mathrm{TAH}^{\star *} \\
\left(\mathrm{t} \mathrm{ha}^{-1}\right)\end{array}$ \\
\hline C & $19.1 \mathrm{a}$ & $17.7 \mathrm{a}$ & $92.7 \mathrm{a}$ & $107.3 a b$ & $12.0 \mathrm{a}$ & $12.9 \mathrm{ab}$ \\
\hline AV1 & $18.7 \mathrm{a}$ & $17.4 \mathrm{a}$ & $93.0 \mathrm{a}$ & $131.1 \mathrm{a}$ & $11.8 \mathrm{a}$ & $15.5 \mathrm{a}$ \\
\hline AV2 & $18.9 \mathrm{a}$ & $17.5 \mathrm{a}$ & $92.6 \mathrm{a}$ & $93.35 \mathrm{~b}$ & $11.9 \mathrm{a}$ & $11.1 \mathrm{~b}$ \\
\hline$A V 1+R$ & $19.0 \mathrm{a}$ & $18.1 \mathrm{a}$ & $95.3 \mathrm{a}$ & 123.7 a & $12.4 \mathrm{a}$ & $15.3 \mathrm{a}$ \\
\hline$A V 2+R$ & $18.9 \mathrm{a}$ & $17.8 \mathrm{a}$ & $94.2 \mathrm{a}$ & $102.7 a b$ & $12.1 \mathrm{a}$ & $12.5 \mathrm{ab}$ \\
\hline
\end{tabular}

*Evaluación durante el mes 11. ** Evaluación durante el mes 13.

Se realizó prueba de Tukey $(p<0.005)$.

Por su parte, la productividad medida en $\mathrm{TCH}$ si marcó diferencias significativas $(p<0.05)$ entre tratamientos, destacándose AV1 y AV1+R con 131.1 y $123.7 \mathrm{t} \mathrm{ha}^{-1}$ respectivamente. Los valores de $\mathrm{TCH}$ en los tratamientos $\mathrm{C}$, AV2 y AV2+R fueron inferiores a la media regional para la variedad CC 85-92, estimada en cerca de $112 \mathrm{t} \mathrm{ha}^{-1}$ (Besosa, 2006; Microfertiza, 2008; CENICAÑA, 2014).

De manera similar al contenido de sólidos, la variable rendimiento tampoco varió entre tratamientos. La siembra intercalada de frijol caupí como AV en caña de azúcar, no incide específicamente en la concentración de azúcares en los tallos, que está más influenciada por aspectos fisiológicos ligados a la fotosíntesis, la humedad del suelo y la técnica de maduración empleada (Subirós, 2000). En el Valle del Cauca, el rendimiento promedio para la variedad CC 85-92 es de 11.5\% (CENICAÑA, 2014), similar a los valores obtenidos en este ensayo.

A nivel económico, la variable TAH es muy importante ya que de ella dependen en buena medida los ingresos monetarios del sector azucarero. El promedio regional de TAH para la variedad CC 85-92 es de $12.9 \mathrm{t} \mathrm{ha}^{-1}$ (CENICAÑA, 2014). En el 
ensayo, los tratamientos $\mathrm{AV} 1$ y $\mathrm{AV}+\mathrm{R}$ fueron significativamente $(p<0.05)$ mayores a los demás, con valores de TAH de 15.5 y $15.3 \mathrm{t} \mathrm{ha}^{-1}$, respectivamente. La aplicación de la cepa Rhizobium sp. en $\mathrm{AV} 1+\mathrm{R}$, generaría un costo adicional sin beneficios económicos en la producción de azúcar. Por su parte, el AV1 incrementaría los ingresos económicos percibidos por productividad de azúcar en cerca de $20 \%$, con respecto al tratamiento control y al promedio regional, siendo un aspecto importante para la sustentabilidad del sector.

\section{Conclusiones}

Bajo las condiciones en las que se desarrolló este ensayo, el AV frijol caupí Vigna unguiculata L., aportó cantidades considerables de materia orgánica al suelo, redujo las poblaciones de arvenses e incrementó la productividad de azúcar cuando se sembró en surcos 1x1 con caña de azúcar, convirtiéndose en alternativa tecnológica viable a favor de la sustentabilidad del sector azucarero.

\section{Agradecimientos}

Los autores presentan sus agradecimientos al Programa Semilleros de Investigación de la Universidad Nacional Abierta y a Distancia UNAD, al Ingenio azucarero RíoPaila-Castilla S.A. y al Centro de Investigación de la Caña de Azúcar CENICAÑA.

\section{Literatura citada}

1. ASOCAÑA. (2008). Asumiendo los retos con energía. Informe anual 2007-2008. Cali. 1, 51.

2. Berlingeri, C., Yuncosa, N. \& Pérez, T. (2008). Evaluación de cinco leguminosas en barbecho mejorado para el control de malezas en la planicie del río Motatán, Estado Trujillo, Venezuela. Revista Agronomía Tropical. Vol. 58(2): 117- 123.

3. Besosa, R. (2006). Respuesta de la caña de azúcar, variedad CC 85-92, a la fertilización con elementos menores. Memorias VII Congreso de Tecnicaña. Cali. p. 112-117.

4. CENICAÑA. (1995). El cultivo de la caña en la zona azucarera de Colombia. Cali. CENICAÑNA, Vol. 1, 31-62.
5. CENICAÑA. (2014). Centro de Investigación de la Caña de Azúcar de Colombia. Informe anual 2014. Recuperado de: http://www.cenicana.org/web2/index.php/publicaciones

6. Cuero, R. (2012). Hacia un sistema complementario de producción más limpia en suelos degradados por salinidad. Revista Ambiente y Sostenibilidad. Vol. 2: 59-68.

7. Da costa, M.B., Calegari, A., Mondardo, A., Bulisani, E.A., Wildner, L., Alcântara, P., Miyasaka, S. \& Amado, T. J. (1993). Adubação verde no sul do Brasil. Rio de Janeiro, ASPTA. Segunda Edição. 346p.

8. Dávalos, E. (2007). La caña de azúcar: ¿una amarga externalidad? Revista Desarrollo y Sociedad. Vol. 59: 117- 164. Edición electrónica.

9. Delgado, M.J., Casella, S. \& Bedmar, E.J. (2007). Denitrification in Rhizobia-Legume Symbiosis. Chapter 6. In Biology of the Nitrogen Cycle. Edited by H. Bothe, S.J. Ferguson and W.E. Newton. 10p.

10. Duarte, J.M., Pérez, H.E., Pezo, D.A., Arce, J., Romero, F. \& Argel, P.J. (1995). Producción de maíz (Zea mays L.), soya (Glycine max L.) y Caupí (Vigna unguiculata L. Walp) sembrados en asocio con gramíneas del trópico húmedo. Revista Pasturas Tropicales. Vol. 17 (2): 12- 19.

11. Franco, R., Torres, J.S. \& Patoja, J.E. (2009). Impacto de la siembra a $1.75 \mathrm{~m}$ en la productividad de caña de azúcar en el Ingenio Mayagüez. Memorias VIII Congreso de la Asociación Colombiana de Técnicos de la Caña de Azúcar- 2009. Cali. Pp: 298- 302.

12. Gana, A.K. \& Busari, L.D. (2001). Effect of Green Manuring and Farm Yard Manure on Growth and Yield of Sugarcane. National Cereals Research Institute, P.M.B. 8, Bida, Niger State, Nigeria. Sugar Tech. Vol. 3(3): 97- 100.

13. Gómez, J.F. (1995). Control de malezas. En: CENICAÑA. EL cultivo de la caña de azúcar en la zona azucarera de Colombia, Cali, CENICAÑA. pp. 143- 152.

14. Herrera, A. (2011). Estudio comparativo de métodos para la determinación de sacarosa y azúcares reductores en miel virgen de caña utilizados en el ingenio Pichichí S.A. (tesis de pregrado) Universidad Tecnológica de Pereira, Colombia. 131p.

15. Jaramillo, D. (2002). Introducción a la Ciencia del Suelo. Universidad Nacional de Colombia sede Medellín. 619p.

16. Larrahondo, J. \& Villegas, F. (1995). Control y características de maduración. En CENICAÑA. El cultivo de la caña en la zona azucarera de Colombia, Cali, CENICAÑA. pp: 297- 313.

17. López, G I., Fontenot, J. P. \& García, T. (2011). Comparaciones entre cuatro métodos de estimación de biomasa en praderas de Festuca alta. Revista Mexicana de Ciencias Pecuarias. Vol. 2(2): 209- 220.

18. Microfertiza (2008). Producción y calidad de la caña de azúcar con nutrimentos Microfertisa. Revista Tecnicaña. Vol. 12(20): 28- 31.

19. Obando, D.M. (2012). Respuesta fisiológica del frijol Caupí (Vigna unguiculata L.- Walp) a la coinoculación de bacterias diazotróficas de los géneros Azotobacter y Rhizobium en suelos del departamento del Cesar. (tesis de Maestría). Universidad Nacional de Colombia. Bogotá. 78p. 
20. Padilla, C., Colom, S. \& Díaz, M.F. (2001). Efecto del intercalamiento de Vigna unguiculata y Zea maiz en el establecimiento de Leucaena leucocephala vc Perú y Panicum maximum vc likoni. Revista Cubana de Ciencia Agrícola. Vol. 35(2): 167-173.

21. Pérez, M.A., Peña, M.R. \& Álvarez, P. (2011). Agroindustria cañera y uso del agua: Análisis crítico en el contexto de la política de agrocombustibles en Colombia. Revista Ambiente \& Sociedade. Vol. 14(2): 153- 178. Campinas.

22. Prager, M., Sanclemente, O.E., Sánchez de Prager, M., Gallego, J.M. \& Ángel, D.I. (2012). Abonos verdes: Tecnología para el manejo agroecológico de los cultivos. Revista Agroecología, No. 7: 53- 62. Murcia España.
23. Sanclemente, O.E. (2013). Efecto de Mucuna pruriens sobre la actividad simbiótica rizosférica y la movilización de $\mathrm{N}$ y $\mathrm{P}$ en un sistema de cultivo: maíz (Zea mays L.) y soya (Glycine max L.). (tesis doctoral) Universidad Nacional de Colombia, Sede Palmira. 123p.

24. Shindoi, M., Prause, J. \& Jover, P.L. (2012). Descomposición de Vigna unguiculata (caupí) en un Argiudol Típico de Colonia Benítez, Chaco. Revista RIA. Vol. 38(1): 86-90.

25. Subirós, F. (2000). El cultivo de la caña de azúcar. Edit. Eunet. San José de Costa Rica. 448p.

26. Zúñiga, O., Osorio, J.C., Cuero, R. \& Peña, J. (2011). Evaluación de Tecnologías para la Recuperación de Suelos Degradados por Salinidad. Rev. Fac. Nal. Agr. Medellín. Vol. 64(1): 5769- 5779.

\section{Conflicto de Intereses}

Los autores declaran no tener ningún conflicto de intereses 
UNAP 